\title{
Cirurgia em metástase vertebral: Proposta de modelo preditivo de morbimortalidade*
}

\section{Spinal Metastasis Surgery: A Proposal for a Predictive Model of Morbidity and Mortality}

\author{
Pedro Reggiani Anzuatégui ${ }^{1,2}$ Luiz Antônio Munhoz da Cunha ${ }^{1,3}$ Glauco José Pauka Mello 2
}

Edmar Stieven Filho ${ }^{1,3}$ Xavier Soler Graells ${ }^{1}$

\footnotetext{
${ }^{1}$ Serviço de Ortopedia e Traumatologia, Hospital de Clínicas, Universidade Federal do Paraná (UFPR), Curitiba, PR, Brasil

2 Serviço de Ortopedia Oncológica, Hospital Erasto Gaertner, Curitiba, PR, Brasil

${ }^{3}$ Departamento de Cirurgia, Faculdade de Medicina, Universidade

Federal do Paraná (UFPR), Curitiba, PR, Brasil
}

\begin{abstract}
Endereço para correspondência Pedro Reggiani Anzuatégui, MD, MSc, Rua Ari José Valle, 980, casa 16, 82030-025, Curitiba, PR, Brasil (e-mail: pedroanzuategui@hotmail.com).
\end{abstract}

Rev Bras Ortop 2019;54:665-672.

\begin{abstract}
Resumo
Palavras-chave

- coluna vertebral/ cirurgia

- comorbidade

- linfócitos

- morbidade

- mortalidade

- metástase neoplásica

- complicações pósoperatórias

Objetivo Desenvolver um modelo preditivo de morbimortalidade pós-operatória precoce com o intuito de auxiliar na seleção dos candidatos à cirurgia para metástase vertebral. Métodos Análise retrospectiva de pacientes consecutivos operados por doença metastática vertebral. As características pré-operatórias consideradas possivelmente prognósticas foram: sexo, idade, comorbidades, velocidade de progressão tumoral e contagem de leucócitos e linfócitos no sangue periférico. Os desfechos pós-operatórios analisados foram: mortalidade em 30 dias e em 90 dias, e presença de complicações. Um modelo preditivo foi desenvolvido a partir de fatores independentemente associados a esses três desfechos. Testou-se então o modelo estabelecido quanto à tendência de prever eventos adversos, à capacidade de discriminação e à calibração. Resultados Um total de 205 pacientes foram operados entre 2002 e 2015. A mortalidade em 30 dias e em 90 dias e a incidência de complicações foram de $17 \%, 42 \%$ e $31 \%$, respectivamente. Os fatores independentemente associados a esses três desfechos, e que constituíram o modelo preditivo, foram: presença de comorbidades, tumor primário de progressão não lenta, e linfócitos abaixo de 1.000 células/ $\mu \mathrm{L}$. A exposição a nenhum, um, dois ou três fatores definiu as categorias do modelo preditivo: baixo, moderado, alto e de extremo risco, respectivamente. Comparando-se as categorias, houve aumento progressivo na ocorrência dos desfechos, seguindo tendência linear. A capacidade de discriminação foi de $72 \%, 73 \%$ e $70 \%$ para mortalidade em 30 dias, em 90 dias e incidência de complicações, respectivamente. Não ocorreu falta de calibração.
\end{abstract}

Trabalho desenvolvido no Hospital Erasto Gaertner pelo Programa de Pós-Graduação em Clínica Cirúrgica da Universidade Federal do Paraná (UFPR), Curitiba, PR, Brasil.

recebido

07 de Junho de 2018

aceito

06 de Agosto de 2018
DOI https://doi.org/

10.1055/s-0039-1697018. ISSN 0102-3616.
Copyright $\odot 2019$ by Sociedade Brasileira License terms de Ortopedia e Traumatologia. Published by Thieme Revinter Publicações Ltda, Rio de Janeiro, Brazil 
Conclusão $\mathrm{O}$ modelo preditivo permite estimar a morbimortalidade após a cirurgia para metástase vertebral e hierarquizar os riscos em baixo, moderado, alto e extremo.

Abstract

\section{Keywords}

- spine/surgery

- comorbidity

- lymphocytes

- morbidity

- mortality

- neoplasm metastasis

- postoperative complications
Objective To develop a predictive model of early postoperative morbidity and mortality with the purpose of assisting in the selection of the candidates for spinal metastasis surgery. Methods A retrospective analysis of consecutive patients operated for metastatic spine disease. The possible prognostic preoperative characteristics were gender, age, comorbidities, tumor growth rate, and leukocyte and lymphocyte count in the peripheral blood. The postoperative outcomes were 30-day mortality, 90-day mortality and presence of complications. A predictive model was developed based on factors associated with these three outcomes. The final model was then tested for the tendency to predict adverse events, discrimination capacity and calibration.

Results A total of 205 patients were surgically treated between 2002 and 2015. The rates of the 30-day mortality, 90-day mortality and presence of complications were of $17 \%, 42 \%$ and $31 \%$ respectively. The factors independently associated with these three outcomes, which constituted the predictive model, were presence of comorbidities, no slow-growing primary tumor, and lymphocyte count below 1,000 cells/ $\mu \mathrm{L}$. Exposure to none, one, two or three factors was the criterion for the definition of the following categories of the predictive model: low, moderate, high and extreme risk respectively. Comparing the risk categories, there was a progressive increase in the occurrence of outcomes, following a linear trend. The discrimination capacity was of $72 \%, 73 \%$ and $70 \%$ for the 30 -day mortality, 90-day mortality and complications respectively. No lack of calibration occurred.

Conclusion The predictive model estimates morbidity and mortality after spinal metastasis surgery and hierarchizes risks as low, moderate, high and extreme.

\section{Introdução}

Por estar relacionado a uma alta incidência de complicações pósoperatórias, ${ }^{1}$ o tratamento cirúrgico para metástase vertebral é controverso, e vem sendo debatido no ambiente acadêmico há décadas. $^{2}$ Outras formas de tratamento, como a radioterapia, têm menos efeitos adversos, ${ }^{3}$ e se tornam cada vez mais atraentes, principalmente por se tratar de um grupo de pacientes que, frequentemente, não atinge doze meses de sobrevida. ${ }^{4} \mathrm{O}$ portador de doença metastática vertebral (DMV) tem em média 60 anos de idade, e um estado de saúde fragilizado por comorbidades como imunodepressão e desnutrição. ${ }^{5}$

Nos casos de tratamento cirúrgico da DMV, as complicações pós-operatórias ocorrem em 17-51\% dos casos, ${ }^{1,6}$ e, muitas vezes, interferem negativamente na história natural da doença, abreviando a já curta sobrevida do paciente. Por outro lado, quando se leva em consideração a cirurgia, há evidências científicas dos benefícios desse tratamento, inclusive com resultados superiores aos da radioterapia isolada, ${ }^{7}$ pois possibilita a descompressão intracanal direta das estruturas (ressecção da massa tumoral), e também a estabilização mecânica da coluna vertebral pela fixação cirúrgica. Esses procedimentos podem levar à manutenção/recuperação da função urinária, redução da dor, e recuperação, em alguns casos, da capacidade de deambulação. ${ }^{8-10}$

A antecipação de eventos adversos resultantes da cirurgia em casos de DMV e a previsão da evolução positiva ou negativa dos casos operados por meio de modelos preditivos (MPs) também originam muitas pesquisas. Vários MPs estão disponíveis na literatura, sendo que alguns ajudam na seleção de pacientes, mas a maioria tem como função principal estimar o tempo de sobrevida do paciente. ${ }^{11}$ Os autores não encontraram na literatura atual nenhum MP que ajudasse a estimar a morbimortalidade precoce após o tratamento cirúrgico de DMV. Assim, os objetivos do presente estudo foram avaliar os parâmetros clínicos e laboratoriais que influenciam a morbimortalidade precoce após o tratamento cirúrgico da DMV e determinar, com base na análise multivariada desses parâmetros, um MP que ajude o médico assistente a estimar a morbimortalidade pós-operatória precoce em pacientes com lesões metastáticas vertebrais.

\section{Materiais e Métodos}

Foi realizada uma análise retrospectiva de uma coorte de pacientes operados por DMV entre janeiro de 2002 e 31 de dezembro de 2015. Este estudo foi aprovado pelo Comitê de Ética da instituição onde foi realizado o estudo. Por se tratar de estudo retrospectivo, não houve necessidade de aplicação do Termo de Consentimento Livre e Esclarecido.

\section{Critérios de Inclusão}

(1) Pacientes únicos e consecutivos submetidos a cirurgia aberta; e (2) presença de estudo anatomopatológico confirmando o diagnóstico de neoplasia maligna vertebral metastática. 


\section{Critérios de Exclusão}

(1) Cirurgia primária ou revisão em outra instituição; (2) dados incompletos de prontuário; e (3) perda de seguimento.

\section{Determinação dos fatores de risco possivelmente prognósticos}

Os autores consideraram as seguintes características préoperatórias como possíveis fatores de risco para a ocorrência dos desfechos negativos na DMV:

(1) sexo masculino;

(2) idade $\geq 70$ anos;

(3) presença de pelo menos uma comorbidade da lista do -Quadro 1. As comorbidades listadas foram obtidas pela união das comorbidades significativas propostas por Charlson et al $^{12}$ e Elixhauser et al; ${ }^{13}$

(4) tumor primário considerado de progressão não lenta. Adotou-se o modelo de Tomita et al, ${ }^{14}$ em que são consideradas neoplasias de progressão lenta o câncer de mama, de próstata e de tireoide. Neste grupo, foram também considerados tumores de progressão lenta o mieloma múltiplo e o linfoma;

(5) contagem de leucócitos $\geq 13.000$ células/ $\mu \mathrm{L}$ no sangue periférico; e

(6) contagem de linfócitos $<1.000$ células/ $\mu \mathrm{L}$ no sangue periférico.

\section{Possíveis Desfechos do Tratamento da Doença Metastástica Vertebral Para Elaboração de Modelo Preditivo}

Para a elaboração do MP, foram considerados os seguintes desfechos:

(1) mortalidade em 30 dias de pós-operatório;

(2) mortalidade em 90 dias de pós-operatório; e

(3) incidência de pelo menos uma complicação.

Foram consideradas complicações pós-operatórias aquelas ocorridas em até 30 dias do procedimento, com base na definição da Organização Mundial da Saúde (OMS). ${ }^{15}$ Elas foram caracterizadas e classificadas pelo método de Rampersaud et al, ${ }^{16}$ sendo incluídas apenas as maiores, que foram agrupadas em:

(1) locais/sistêmicas; e

(2) infecciosas/não infecciosas.

Quadro 1 Índice de comorbidades

\section{- Diabetes}

- Doença pulmonar crônica

- Infarto do miocárdio prévio

- Insuficiência cardíaca congestiva

- Arritmia cardíaca

- Doença da circulação pulmonar

- Doença vascular periférica

- Doença vascular cerebral

- Demência

- Insuficiência renal

- Insuficiência hepática

- Doença do tecido conjuntivo

- Coagulopatia

- Paralisia prévia

- Úlcera péptica

- Síndrome da imunodeficiência adquirida

\section{Modelo Preditivo}

A comparação da frequência de ocorrência dos desfechos em indivíduos expostos e não expostos aos possíveis fatores de risco, e a análise multivariada, determinando os fatores com significância estatística e os fatores associados a todos os desfechos, permitiram a hierarquização dos riscos em baixo, moderado, alto e extremo. O MP foi testado com relação à tendência de ocorrência de eventos, à capacidade de discriminação, e à calibração.

\section{Análise Estatística}

Variáveis contínuas foram dicotomizadas e tratadas como variáveis categóricas. Os testes de Fisher e qui-quadrado foram aplicados para a avaliação de risco. A análise da mortalidade em 30 e 90 dias de pós-operatório foi realizada de forma separada para cada ponto no tempo. O método de KaplanMeier foi utilizado para elaboração das curvas de sobrevida. As categorias do MP final foram comparadas quanto à tendência de ocorrência de eventos por meio do teste qui-quadrado. A capacidade de discriminação e a calibração do modelo final foram analisadas por meio da curva da característica de operação do receptor (COR) e do teste de Hosmer-Lemeshow, respectivamente. Modelos de regressão logística foram aplicados aos grupos de variáveis, desde que $p<0,05$ na análise bivariada. O intervalo de confiança foi de $95 \%$ para todas as análises. Os programas de computador R ( $R$ Foundation for Statistical Computing, Viena, Áustria), versão 3.3.1, e MedCalc (MedCalc Software, Ostende, Bélgica), versão 17.6, foram utilizados para a realização dos testes estatísticos. ${ }^{17,18}$

\section{Resultados}

\section{Pacientes}

Foram operados 306 pacientes, e, após a adoção dos critérios de inclusão e exclusão, restaram 205 pacientes que compõem este estudo. Na - Tabela 1, estão apresentadas as características gerais dos pacientes estudados.

\section{Fatores de Risco Possivelmente Prognósticos}

Um total de 114 pacientes (55\%) eram do sexo masculino; 48 pacientes (23\%) apresentavam idade $\geq 70$ anos; 65 pacientes (32\%) apresentavam 1 ou mais comorbidades; 81 pacientes (40\%) eram portadores de tumores de progressão não lenta; 40 pacientes (20\%) apresentavam leucócitos $\geq 13.000$ células/ $\mu \mathrm{L}$ (média de 9.700 células $/ \mu \mathrm{L}$ ); e 51 pacientes (25\%) apresentavam linfócitos $<1.000$ células/ $\mu \mathrm{L}$ (média de 1.600 células $/ \mu \mathrm{L})$.

\section{Possíveis Desfechos do Tratamento da Doença Metastástica Vertebral Para a Elaboração de Modelo Preditivo}

A mortalidade em 30 dias foi de $17 \%(\mathrm{n}=36)$, e em 90 dias, de $43 \%(\mathrm{n}=88)$. A incidência de complicações pós-operatórias foi de 31\% ( $\mathrm{n}=64)$, e está apresentada na - Tabela 2.

\section{Análise Estatística}

As - Tabelas 3 e 4 apresentam estudo analítico de risco com relação aos possíveis fatores preditivos dos desfechos. 
Tabela 1 Características gerais dos 205 pacientes submetidos a cirurgia para metástase vertebral

\begin{tabular}{|c|c|}
\hline Variável & $n(\%)$ \\
\hline Sexo masculino & $114(55 \%)$ \\
\hline Idade (anos), média \pm desvio padrão & $58,9 \pm 13,3$ \\
\hline Óbitos antes da alta & $14(7 \%)$ \\
\hline Vivos durante a coleta dos dados & $12(6 \%)$ \\
\hline \multicolumn{2}{|l|}{ Abordagem } \\
\hline Cervical/cervicotorácica & $11(5 \%)$ \\
\hline Torácica & $70(34 \%)$ \\
\hline Toracolombar & $71(35 \%)$ \\
\hline Lombar/lombossacra & $49(24 \%)$ \\
\hline Múltipla & $4(2 \%)$ \\
\hline Via de acesso posterior & 201 (95\%) \\
\hline \multicolumn{2}{|l|}{ Tumor primário } \\
\hline Próstata & $51(24 \%)$ \\
\hline Mama & $43(21 \%)$ \\
\hline Mieloma múltiplo & $26(13 \%)$ \\
\hline Desconhecido & $20(10 \%)$ \\
\hline Útero & $12(6 \%)$ \\
\hline Outros & $53(25 \%)$ \\
\hline \multicolumn{2}{|l|}{ Comorbidades } \\
\hline Diabetes & $25(12 \%)$ \\
\hline Doença pulmonar crônica & $20(10 \%)$ \\
\hline Insuficiência cardíaca & $7(3 \%)$ \\
\hline Infarto do miocárdio prévio & $5(2 \%)$ \\
\hline Arritmia cardíaca & $4(2 \%)$ \\
\hline Outras & $13(6 \%)$ \\
\hline
\end{tabular}

Detectou-se que as características que se comportam como fator de risco independente para a ocorrência de complicações do tipo sistêmicas são: idade $\geq 70$ anos (razão de probabilidades [RP]: 2,$44 ; p<0,05)$; tumor primário de progressão não lenta (RP: 2,$54 ; p<0,05$ ); e contagem total de linfócitos $<1.000$ células/ $\mu \mathrm{L}$ (RP: 3,$19 ; p<0,01$ ). Tumor primário de progressão não lenta é a única característica pré-operatória associada com infecção de sítio cirúrgico (RP: 2,52; $p<0,05)$. Idade $\geq 70$ anos e tumor primário de progressão não lenta são fatores de risco independentes para complicação do tipo infecciosa (RP: 2,82; $p<0,05$; e RP: 3,$22 ; p<0,01$, respectivamente). Após análise multivariada, a mortalidade advinda de complicação está associada com idade $\geq 70$ anos (RP: 3,$35 ; p<0,01$ ), presença de comorbidades (RP: 2,$74 ; p<0,01$ ), e contagem total de linfócitos $<1.000$ células/ $\mu \mathrm{L}$ (RP: 3,$61 ; p<0,0001$ ). O óbito relacionado a complicações infecciosas, após análise de múltiplas variáveis, tem correlação com idade $\geq 70$ anos (RP: 2,61; $p<0,05$ ), tumor primário de progressão não lenta (RP: 2,82 ; $p<0,05$ ), e contagem total de linfócitos $<1.000$ células $/ \mu \mathrm{L}$ (RP: $4,23 ; p<0,01)$.
Tabela 2 Incidência de complicações após tratamento cirúrgico para metástase vertebral

\begin{tabular}{|l|l|}
\hline Variáveis & $\mathbf{n}(\%)$ \\
\hline Sistêmicas & \\
\hline Pneumonia & $14(6,8)$ \\
\hline Óbito de causa desconhecida & $11(5,4)$ \\
\hline Hemorragia digestiva & $4(2,0)$ \\
\hline Insuficiência respiratória & $3(1,5)$ \\
\hline Insuficiência renal & $2(1,0)$ \\
\hline Sepse de foco urinário & $1(0,5)$ \\
\hline Sepse de foco desconhecido & $1(0,5)$ \\
\hline Outras & $4(2,0)$ \\
\hline Subtotal & $40(19,5)$ \\
\hline Complicações Locais & \\
\hline Infecção de ferida & $20(9,8)$ \\
\hline Deiscência & $2(1,0)$ \\
\hline Hematoma & $1(0,5)$ \\
\hline Piora neurológica & $1(0,5)$ \\
\hline Subtotal & $24(11,7)$ \\
\hline Infecciosas & $36(17,5)$ \\
\hline Não infecciosas & $28(13,7)$ \\
\hline Grau III & $19(9,3)$ \\
\hline Grau IV & $45(21,9)$ \\
\hline Total & $64(31,2)$ \\
\hline
\end{tabular}

Nota: Segundo Rampersaud et al, ${ }^{16}$ a complicação de grau III requer tratamento significativo (cirurgia inesperada ou reinternação, por exemplo), aumentando o tempo de permanência hospitalar por mais de 7 dias e/ou causando sequela por mais de 6 meses. A complicação grau IV é aquela que resulta em óbito.

\section{Modelo Preditivo}

$\mathrm{Na}$ - Tabela 4, estão explicitados os fatores de risco independentes para a ocorrência dos desfechos desta pesquisa. Aqueles que apresentaram significância estatística para os três desfechos foram incluídos no MP, que está ilustrado no -Quadro 2.

As - Figuras 1, 2 e 3 mostram as curvas de sobrevida em 90 dias de pós-operatório, de acordo com as características utilizadas para desenvolver o MP final. A exposição a nenhum, um, dois ou três fatores foi o critério que definiu

Quadro 2 Modelo preditivo

\begin{tabular}{|l|l|l|}
\hline Fatores de risco & $\begin{array}{l}\text { Fatores } \\
\text { presentes }\end{array}$ & $\begin{array}{l}\text { Categoria } \\
\text { de risco }\end{array}$ \\
\hline - Presença de pelo menos & 0 & Baixo \\
\cline { 2 - 3 } $\begin{array}{l}\text { - Tuma comorbidade } \\
\text { progressão não lenta } \\
\text { - Contagem total de } \\
\text { linfócitos no sangue } \\
\text { periférico abaixo de } \\
1.000 \text { células/ } \mu \mathrm{L}\end{array}$ & 1 & Moderado \\
\cline { 2 - 3 } & 2 & Alto \\
\cline { 2 - 3 } & 3 & Extremo \\
\hline
\end{tabular}


Tabela 3 Análise bivariada de características pré-operatórias como possíveis fatores prognósticos de morbimortalidade precoce após tratamento cirúrgico para metástase vertebral

\begin{tabular}{|c|c|c|c|c|}
\hline Característica & $n(\%)$ & $\begin{array}{l}\text { Razão de probabilidades } \\
\text { para mortalidade } \\
\text { em } 30 \text { dias (IC) }\end{array}$ & $\begin{array}{l}\text { Razão de probabilidades } \\
\text { para mortalidade } \\
\text { em } 90 \text { dias (IC) }\end{array}$ & $\begin{array}{l}\text { Razão de probabilidades } \\
\text { para incidência de } \\
\text { complicação (IC) }\end{array}$ \\
\hline \multicolumn{5}{|l|}{ Sexo } \\
\hline Feminino & 91 & Ref. & Ref. & Ref. \\
\hline Masculino & 114 & $1,00^{*}(0,48-2,06)$ & $1,05^{*}(0,60-1,83)$ & $1,15^{*}(0,63-2,10)$ \\
\hline \multicolumn{5}{|l|}{ Idade (anos) } \\
\hline$<70$ & 157 & Ref. & Ref. & Ref. \\
\hline$\geq 70$ & 48 & $2,94^{* * *}(1,37-6,31)$ & $2,08^{* *}(1,08-4.00)$ & $3,13^{* * * *}(1,60-6,14)$ \\
\hline \multicolumn{5}{|l|}{ Comorbidades } \\
\hline Ausente & 140 & Ref. & Ref. & Ref. \\
\hline Presente & 65 & $2,60^{* * *}(1,24-5,41)$ & $2,87^{* * * *}(1,57-5,27)$ & $2,61^{* * *}(1,40-4,88)$ \\
\hline \multicolumn{5}{|c|}{$\begin{array}{l}\text { Tumor primário de } \\
\text { progressão lenta }\end{array}$} \\
\hline Sim & 124 & Ref. & Ref. & Ref. \\
\hline Não & 81 & $2,21^{* *}(1,07-4,59)$ & $3,79^{* * * *}(2,10-6,85)$ & $2,48^{* * *}(1,35-4,56)$ \\
\hline \multicolumn{5}{|l|}{ Leucócitos ( $\mu \mathrm{L})$} \\
\hline$<13.000$ & 165 & Ref. & Ref. & Ref. \\
\hline$\geq 13.000$ & 40 & $1,78^{*}(0,77-4,08)$ & $3,17^{* * *}(1,54-6,52)$ & $1,81^{*}(0,88-3,74)$ \\
\hline \multicolumn{5}{|l|}{ Linfócitos ( $\mu \mathrm{L})$} \\
\hline$\geq 1.000$ & 154 & Ref. & Ref. & Ref. \\
\hline$<1.000$ & 51 & $3,06^{* * *}(1,44-6,52)$ & $1,96^{* *}(1,03-3,72)$ & $2,71^{* * *}(1,40-5,25)$ \\
\hline
\end{tabular}

Abreviaturas: IC, intervalo de confiança de $95 \%$; Ref., variável de referência.

Nota: Valores de $p:{ }^{*}$ se $p>0,05 ;{ }^{* *}$ se $p$ entre 0,05 e 0,$01 ;{ }^{* * *}$ se $p$ entre 0,01 e 0,$001 ;{ }^{* * * *}$ se $p<0,001$.

Tabela 4 Análise multivariada de características pré-operatórias como possíveis fatores prognósticos de morbimortalidade precoce após tratamento cirúrgico para metástase vertebral

\begin{tabular}{|c|c|c|c|c|}
\hline Característica & n (\%) & $\begin{array}{l}\text { Razão de probabilidades } \\
\text { para mortalidade } \\
\text { em } 30 \text { dias (IC) }\end{array}$ & $\begin{array}{l}\text { Razão de probabilidades } \\
\text { para mortalidade } \\
\text { em } 90 \text { dias (IC) }\end{array}$ & $\begin{array}{l}\text { Razão de probabilidades } \\
\text { para incidência de } \\
\text { complicação (IC) }\end{array}$ \\
\hline \multicolumn{5}{|l|}{ Idade (anos) } \\
\hline$<70$ & 157 & Ref. & Ref. & Ref. \\
\hline$\geq 70$ & 48 & $2,73^{* *}(1,20-6,20)$ & $2,06^{*}(0,98-4,36)$ & $3,15^{* * *}(1,51-6,59)$ \\
\hline \multicolumn{5}{|l|}{ Comorbidades } \\
\hline Ausente & 140 & Ref. & Ref. & Ref. \\
\hline Presente $^{\dagger}$ & 65 & $2,33^{* *}(1,07-5,07)$ & $2,60^{* * *}(1,33-5,12)$ & $2,37^{* *}(1,21-4,65)$ \\
\hline \multicolumn{5}{|c|}{$\begin{array}{l}\text { Tumor primário } \\
\text { de progressão lenta }\end{array}$} \\
\hline Sim & 124 & Ref. & Ref. & Ref. \\
\hline Não ${ }^{\dagger}$ & 81 & $2,56^{* *}(1,17-5,62)$ & $4,30^{* * * *}(2,23-8,30)$ & $3,07^{* * *}(1,56-6,04)$ \\
\hline \multicolumn{5}{|l|}{ Leucócitos ( $\mu \mathrm{L})$} \\
\hline$<13.000$ & 165 & Ref. & Ref. & Ref. \\
\hline$\geq 13.000$ & 40 & -- & $2,94^{* *}(1,29-6,70)$ & -- \\
\hline \multicolumn{5}{|l|}{ Linfócitos $(\mu \mathrm{L})$} \\
\hline$\geq 1.000$ & 154 & Ref. & Ref. & Ref. \\
\hline$<1.000^{\dagger}$ & 51 & $3,07^{* * *}(1,37-6,87)$ & $2,19^{* *}(1,06-4,51)$ & $2,84^{* * *}(1,37-5,85)$ \\
\hline
\end{tabular}

Abreviaturas: IC, intervalo de confiança de 95\%; Ref., variável de referência.

Notas: ${ }^{\dagger}$ Característica incluída no modelo preditivo (MP) pela associação com os três desfechos. Valores de $p:{ }^{*}$ se $p>0,05 ;{ }^{* *}$ se $p$ entre 0,05 e 0,01 ; ${ }^{* * *}$ se $p$ entre 0,01 e 0,$001 ;{ }^{* * *}$ se $p<0,001$. 


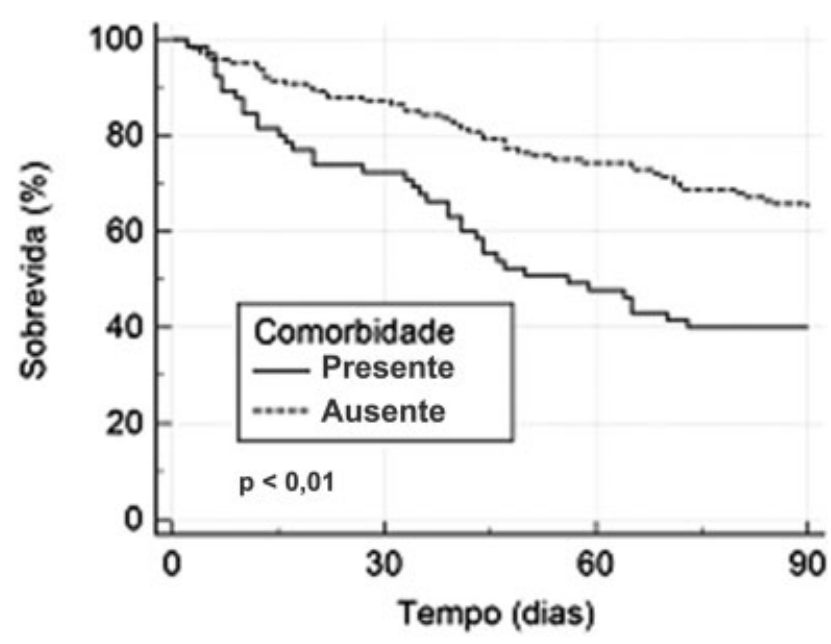

Fig. 1 Sobrevida em 90 dias de pós-operatório de acordo com a presença de comorbidades.

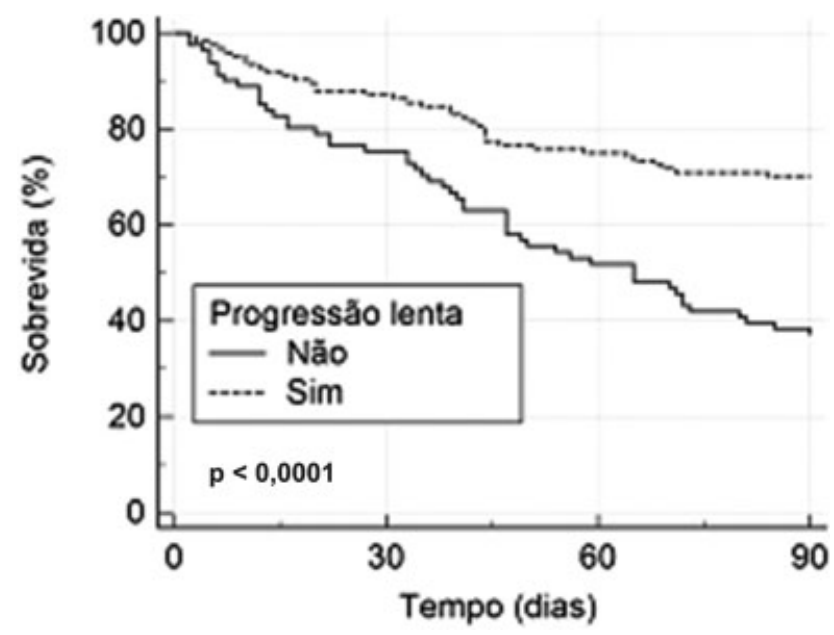

Fig. 2 Sobrevida em 90 dias de pós-operatório de acordo com a velocidade de progressão tumoral.

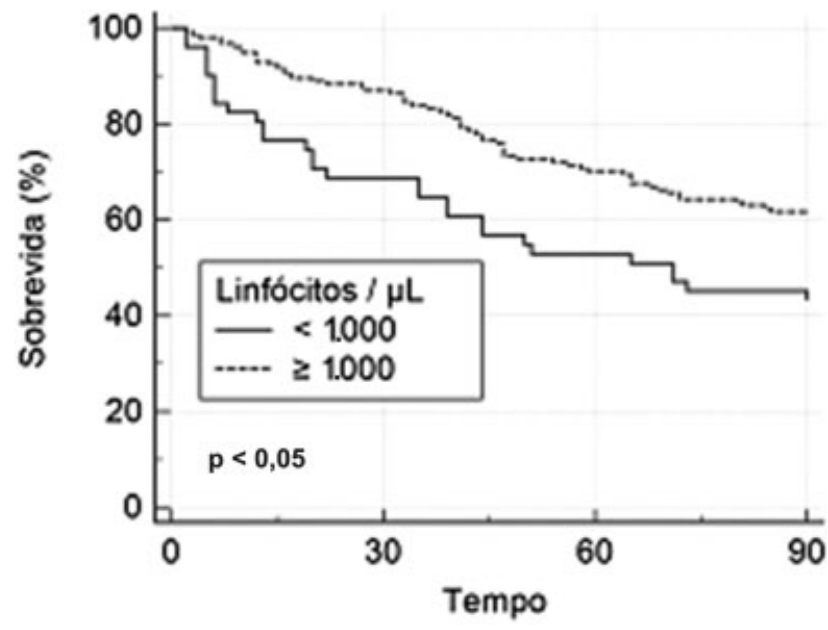

Fig. 3 Sobrevida em 90 dias de pós-operatório de acordo com a contagem pré-operatória de linfócitos no sangue periférico.

as categorias de baixo, moderado, alto e extremo risco, respectivamente. A - Figura 4 ilustra a incidência de morbimortalidade precoce de acordo com cada categoria de risco do MP. A - Figura 5 mostra a sobrevida em 90 dias de pósoperatório, de acordo com as 4 categorias de risco. Comparando-se as categorias do menor para o maior risco, houve aumento progressivo na ocorrência dos desfechos, seguindo tendência linear $(p<0,0001)$. O mesmo ocorreu quando analisadas as complicações sistêmicas $(p<0,0001)$, infecciosas $(p<0,0001)$, óbito por complicação $(p<0,0001)$, óbito por complicação infecciosa $(p<0,0001)$ e infecção de ferida operatória $(p<0,05)$. A capacidade de discriminação do modelo, segundo a curva COR, foi de $72 \%$ para mortalidade em 30 dias, de 73\% para mortalidade em 90 dias, e de $70 \%$ para incidência de complicações. Não houve evidência de falta de calibração pelo teste de Hosmer-Lemeshow.

\section{Discussão}

As complicações da DMV em relação a desfechos cirúrgicos desfavoráveis, incluindo o óbito, que é o pior deles, em nada se comparam às obtidas no tratamento cirúrgico da maioria das doenças ortopédicas. Na cirurgia da DMV, a mortalidade em 90 dias é importante, e a maioria dos autores concorda que esse intervalo de tempo seja o mínimo previsto para se indicar um procedimento altamente mórbido e paliativo. ${ }^{19}$ No entanto, há poucos estudos que abordam esse ponto de corte na sobrevida pós-operatória. ${ }^{20}$

Supõe-se que características clínicas pré-operatórias exerçam maior influência nos resultados cirúrgicos precoces quando comparados com resultados de longo prazo. Os MPs tradicionais, como o sistema de pontuação de Tokuhashi, ${ }^{21}$ focam mais em características que se mostram associadas a resultados cirúrgicos de médio e longo prazo, como a presença de metástases viscerais. Talvez por isso, o escore de Tokuhashi só estime eventos a partir de 180 dias do procedimento. Mais recentemente, Schoenfeld et $\mathrm{al}^{20}$ apontam o nível sérico de albumina como um forte fator de risco para mortalidade em 30 dias, superando até mesmo a velocidade de progressão tumoral. 0 presente estudo, com a esperança de identificar fatores prognósticos mais significativos, e que possam alterar positivamente os desfechos em até três meses do procedimento, avalia algumas características clínicas menos valorizadas em estudos prévios, tais como os índices de comorbidades e a contagem celular no sangue periférico.

Os índices de comorbidades são abordados em raras pesquisas sobre DMV. Patil et al $^{1}$ relatam risco aumentado em 50\% de complicações da cirurgia em DMV em pacientes que apresentam duas comorbidades, de acordo com as citadas por Elixhauser et al. ${ }^{13}$ Arrigo et al $^{22}$ observam risco aumentado em até cinco vezes em pacientes com duas ou mais comorbidades citadas por Charlson et al. ${ }^{12} \mathrm{O}$ presente trabalho mostra que a presença de pelo menos uma comorbidade entre aquelas obtidas pela união das citadas por Charlson et $\mathrm{al}^{12}$ e Elixhauser et $\mathrm{al}^{13}$ representa um fator de risco independente para morbimortalidade precoce após cirurgia em metástase vertebral. Esse é um fator de risco não relatado anteriormente na literatura.

A linfocitopenia secundária pode ter diversas etiologias, entre elas desnutrição, infecção, uso de corticosteroides, radioterapia e quimioterapia. Essas condições são frequentes 


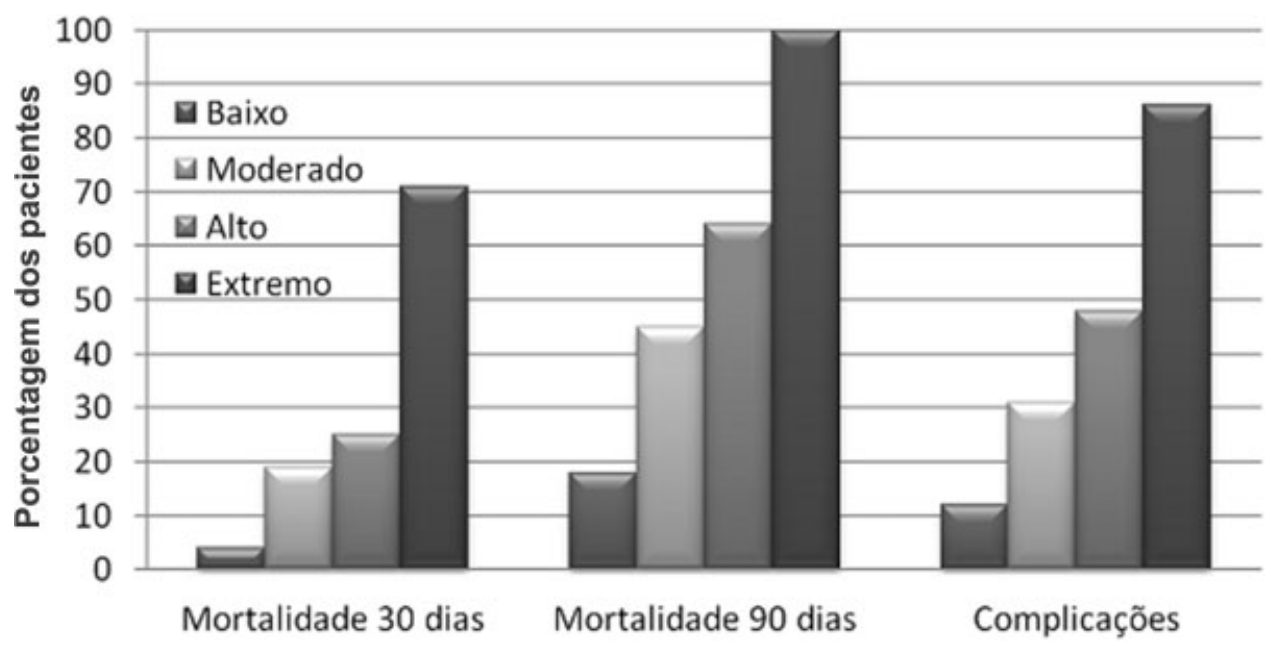

Fig. 4 Morbimortalidade precoce após tratamento cirúrgico para metástase vertebral, de acordo com o modelo preditivo (MP) proposto.

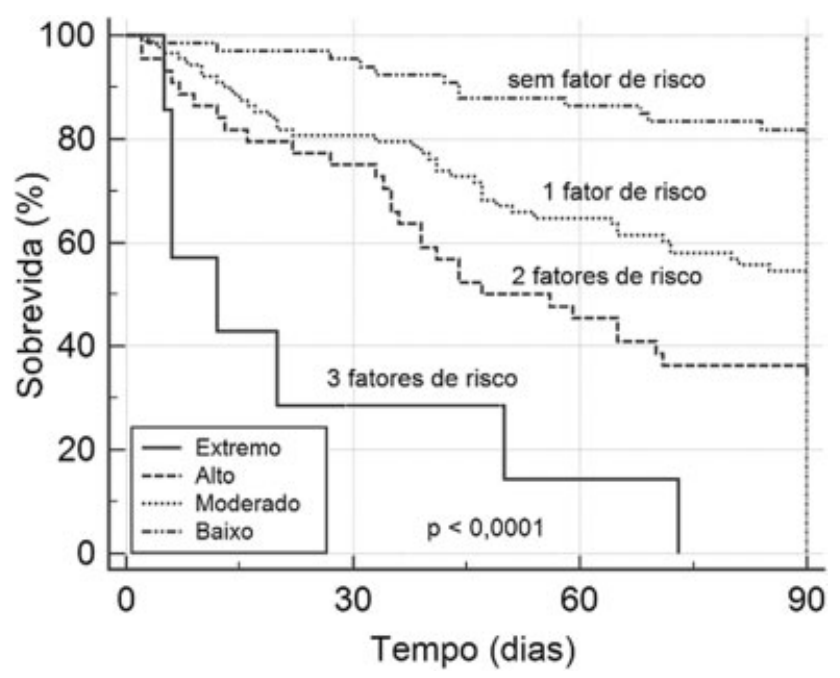

Fig. 5 Sobrevida em 90 dias de pós-operatório de acordo com o modelo preditivo (MP) proposto.

na DMV. A linfocitopenia reduz a ação dos linfócitos B, T e dos exterminadores naturais contra bactérias, vírus e fungos, deixando o organismo suscetível a infecções locais ou à distância. O zinco e algumas vitaminas têm um papel na maturação celular, e a sua carência pode explicar em parte a linfocitopenia apresentada pelos pacientes desnutridos. ${ }^{23}$ Embora a baixa contagem de linfócitos seja um antigo marcador nutricional ${ }^{24}$ e um conhecido fator de mau prognóstico no câncer, ${ }^{25}$ surpreendentemente, a revisão da literatura não mostra a linfocitopenia pré-operatória como fator de risco para cirurgia em DMV. Os trabalhos revisados utilizam ponto de corte diferente para contagem celular $(1.500 / \mu \mathrm{L})$, o que pode explicar os achados conflitantes. ${ }^{5,26}$ Por outro lado, na atual pesquisa, a contagem total de linfócitos $<1.000 / \mu \mathrm{L}$ se mostrou um forte fator de risco. A presença deste dado representa um fator de aumento significativo na ocorrência precoce de complicações e óbito. Ele esteve relacionado a um risco aumentado em quase cinco vezes para a ocorrência de óbito advindo de complicação infecciosa. Nesta série, uma paciente com contagem total de linfócitos pré-operatória de $245 / \mu \mathrm{L}$ foi a óbito em decorrên- cia de sepse por Candida sp, um raro agente causador de infecção sistêmica.

Estudos prévios apontaram que pacientes idosos apresentam piores resultados cirúrgicos em DMV. ${ }^{26,27}$ Neste estudo, identificou-se que indivíduos com 70 anos ou mais têm risco aumentado em 2,73 vezes de mortalidade em 30 dias; em 3,15 vezes de complicações totais; em 2,44 vezes de complicações sistêmicas; em 2,82 vezes de complicações infecciosas; em 3,35 vezes na incidência de óbito por complicação; e em 2,61 vezes na incidência de óbito por complicação infecciosa. No entanto, por falhar na previsão de mortalidade em 90 dias, essa característica foi excluída do MP final.

Não surpreendem os resultados encontrados sobre a influência da agressividade do tumor primário em relação à incidência de complicações e à mortalidade em 30 e 90 dias da cirurgia. Diversos estudos pregressos relatam pior prognóstico em grupos de pacientes portadores de tumores com tipos histológicos mais agressivos. ${ }^{26}$

Neste trabalho, foi proposto um MP para estimar morbimortalidade precoce em DMV que leva em consideração não somente a agressividade do tumor primário, como também a condição sistêmica do paciente (—Quadro 2). Laufer et al, ${ }^{28} \mathrm{em}$ seu algoritmo para conduta, afirmam que a condição sistêmica do paciente é fator decisivo na tomada de decisão cirúrgica. No entanto, nenhum dos muitos MPs existentes na literatura considera a presença de comorbidades e a capacidade imunológica do paciente. ${ }^{11}$ Apenas Ghori et $\mathrm{al}^{29}$ fazem referência à influência no estado nutricional analisando a albumina sérica, e sugerem esse fator como uma possível ferramenta estimadora de complicações.

O MP proposto estima, por categoria, a ocorrência de eventos desfavoráveis em 90 dias da cirurgia. Neste trabalho, os pacientes de baixo risco apresentaram as menores taxas de morbimortalidade pós-operatória, enquanto os pacientes na categoria de extremo risco apresentaram os piores resultados ( - Figuras 4 e 5). Pesquisas futuras poderiam esclarecer se este MP é útil para orientar a decisão terapêutica. Acredita-se que, devido à simplicidade de aplicação, este MP poderia ser uma das primeiras ferramentas utilizadas na avaliação do paciente com DMV. 
Esta pesquisa tem diversas limitações, e, sem dúvida, necessita validação futura, principalmente em relação aos resultados do MP. Pelo desenho retrospectivo, pode ter ocorrido viés de seleção, de aferição e de suscetibilidade. Os resultados podem não ser generalizáveis pelo fato de o estudo ter sido realizado em uma única instituição e sobre uma amostra tipicamente heterogênea.

\section{Conclusões}

Os fatores pré-operatórios que permitem predizer a morbimortalidade precoce em casos de DMV são: idade $\geq 70$ anos; presença de pelo menos uma comorbidade de índice específico; tumor primário de progressão não lenta; leucócitos $\geq$ e 13.000 células/ $/ \mathrm{L}$; e contagem total de linfócitos $<1.000$ células $/ \mu \mathrm{L}$. OMP proposto permite estimar a morbimortalidade da cirurgia na DMV e hierarquizar os riscos cirúrgicos em baixo, moderado, alto e extremo.

\section{Conflitos de Interesse}

Os autores declaram não haver conflitos de interesse.

\section{Referências}

1 Patil CG, Lad SP, Santarelli J, Boakye M. National inpatient complications and outcomes after surgery for spinal metastasis from 1993-2002. Cancer 2007;110(03):625-630

2 Cohen J, Alan N, Zhou J, Kojo Hamilton D. The 100 most cited articles in metastatic spine disease. Neurosurg Focus 2016;41(02):E10

3 Rades D, Huttenlocher S, Dunst J, et al. Matched pair analysis comparing surgery followed by radiotherapy and radiotherapy alone for metastatic spinal cord compression. J Clin Oncol 2010;28 (22):3597-3604

4 Finkelstein JA, Zaveri G, Wai E, Vidmar M, Kreder H, Chow E. A population-based study of surgery for spinal metastases. Survival rates and complications. J Bone Joint Surg Br 2003;85(07):1045-1050

5 Wise JJ, Fischgrund JS, Herkowitz HN, Montgomery D, Kurz LT. Complication, survival rates, and risk factors of surgery for metastatic disease of the spine. Spine 1999;24(18):1943-1951

6 Schoenfeld AJ, Le HV, Marjoua Y, et al. Assessing the utility of a clinical prediction score regarding 30-day morbidity and mortality following metastatic spinal surgery: the New England Spinal Metastasis Score (NESMS). Spine J 2016;16(04):482-490

7 Patchell RA, Tibbs PA, Regine WF, et al. Direct decompressive surgical resection in the treatment of spinal cord compression caused by metastatic cancer: a randomised trial. Lancet 2005;366 (9486):643-648

8 Choi D, Fox Z, Albert T, et al. Rapid improvements in pain and quality of life are sustained after surgery for spinal metastases in a large prospective cohort. Br J Neurosurg 2016;30(03):337-344

9 Ibrahim A, Crockard A, Antonietti P, et al. Does spinal surgery improve the quality of life for those with extradural (spinal) osseous metastases? An international multicenter prospective observational study of 223 patients. Invited submission from the Joint Section Meeting on Disorders of the Spine and Peripheral Nerves, March 2007. J Neurosurg Spine 2008;8(03):271-278

10 Quan GM, Vital JM, Aurouer N, et al. Surgery improves pain, function and quality of life in patients with spinal metastases: a prospective study on 118 patients. Eur Spine J 2011;20(11): 1970-1978

11 Tokuhashi Y, Uei H, Oshima M, Ajiro Y. Scoring system for prediction of metastatic spine tumor prognosis. World J Orthop 2014;5(03):262-271

12 Charlson ME, Pompei P, Ales KL, MacKenzie CR. A new method of classifying prognostic comorbidity in longitudinal studies: development and validation. JChronic Dis 1987;40(05):373-383

13 Elixhauser A, Steiner C, Harris DR, Coffey RM. Comorbidity measures for use with administrative data. Med Care 1998;36 (01):8-27

14 Tomita K, Kawahara N, Kobayashi T, Yoshida A, Murakami H, Akamaru T. Surgical strategy for spinal metastases. Spine 2001;26 (03):298-306

15 Haynes AB, Weiser TG, Berry WR, et al; Safe Surgery Saves Lives Study Group. A surgical safety checklist to reduce morbidity and mortality in a global population. N Engl J Med 2009;360(05): 491-499

16 Rampersaud YR, Moro ER, Neary MA, et al. Intraoperative adverse events and related postoperative complications in spine surgery: implications for enhancing patient safety founded on evidencebased protocols. Spine 2006;31(13):1503-1510

$17 \mathrm{R}$ Core Team. RA language and Environment for Statistical Computing. Vienna, Austria: R Foundation for Statistical Computing; 2016

18 BVBA. MedCalc Software. Ostende, Bélgica2017

19 George R, Jeba J, Ramkumar G, Chacko AG, Leng M, Tharyan P. Interventions for the treatment of metastatic extradural spinal cord compression in adults. Cochrane Database Syst Rev 2008; (04):CD006716

20 Schoenfeld AJ, Leonard DA, Saadat E, Bono CM, Harris MB, Ferrone ML. Predictors of 30- and 90-Day Survival Following Surgical Intervention for Spinal Metastases: A Prognostic Study Conducted at Four Academic Centers. Spine 2016;41(08):E503-E509

21 Tokuhashi Y, Matsuzaki H, Oda H, Oshima M, Ryu J. A revised scoring system for preoperative evaluation of metastatic spine tumor prognosis. Spine 2005;30(19):2186-2191

22 Arrigo RT, Kalanithi P, Cheng I, et al. Charlson score is a robust predictor of 30-day complications following spinal metastasis surgery. Spine 2011;36(19):E1274-E1280

23 Brass D, McKay P, Scott F. Investigating an incidental finding of lymphopenia. BMJ 2014;348:g1721

24 Bharadwaj S, Ginoya S, Tandon P, et al. Malnutrition: laboratory markers vs nutritional assessment. Gastroenterol Rep (Oxf) 2016; 4(04):272-280

25 Trédan O, Ray-Coquard I, Chvetzoff G, et al. Validation of prognostic scores for survival in cancer patients beyond first-line therapy. BMC Cancer 2011;11:95

26 Luksanapruksa P, Buchowski JM, Hotchkiss W, Tongsai S, Wilartratsami S, Chotivichit A. Prognostic factors in patients with spinal metastasis: a systematic review and meta-analysis. Spine J 2017; 17(05):689-708

27 Chi JH, Gokaslan Z, McCormick P, Tibbs PA, Kryscio RJ, Patchell RA. Selecting treatment for patients with malignant epidural spinal cord compression-does age matter?: results from a randomized clinical trial Spine 2009;34(05):431-435

28 Laufer I, Rubin DG, Lis E, et al. The NOMS framework: approach to the treatment of spinal metastatic tumors. Oncologist 2013;18 (06):744-751

29 Ghori AK, Leonard DA, Schoenfeld AJ, et al. Modeling 1-year survival after surgery on the metastatic spine. Spine J 2015;15 (11):2345-2350 